\title{
Trials for oxide photo-thermoelectrics
}

\author{
Ichiro Terasaki • Ryuji Okazaki • \\ Partha Sarathi Mondal · Yu-Chin Hsieh
}

Received: 18 February 2014/ Accepted: 4 April 2014/Published online: 1 May 2014

(C) The Author(s) 2014. This article is published with open access at Springerlink.com

\begin{abstract}
Thermoelectrics is an energy conversion technology from heat into electricity, and vice versa, through the thermoelectric phenomena in solids, while photovoltaics is an energy conversion technology from solar photon energy into electricity using the photo-excitations in solids. We are trying to find a way to combine thermoelectrics with photovoltaics to establish a new method to generate renewable energy with high efficiency. In this article, we show two approaches for this purpose using oxide materials: thermoelectric energy conversion by photo-excited carriers and the thermoelectric power generation using a focused light as a heat source.
\end{abstract}

Keywords Thermoelectric power generation - Oxide . Photo-doping · Photo-thermoelectrics

\section{Introduction}

No one can deny that our modern society is based on vast consumption of electric/chemical energies. Since all the developing countries have the right to enjoy life as comfortable as the advanced countries do, energy demands are increasing year by year in spite of serious shortage of petroleum. Thus, a search for sufficient energy resources is a responsibility of researchers in all areas of science and technology. Best energy resources are of no doubt

This work was partially supported by ALCA, Japan Science and Technology Agency, and by The Mitsubishi Foundation.

I. Terasaki $(\bowtie) \cdot$ R. Okazaki · P. S. Mondal · Y.-C. Hsieh Department of Physics, Nagoya University, Nagoya 464-8602, Japan

e-mail: terra@cc.nagoya-u.ac.jp renewable energies, which preliminarily come from the solar energy.

The energy conversion technique using the solar energy is classified into two; The one is photovoltaics in which an electron-hole pair created by an incident photon is separated by an internal electric field at the $p-n$ junction [27]. This technology is now commercially available as solar battery cells. The other is solar-thermal energy conversion, where heat generated by focused sunlight vaporizes water to rotate a gas turbine [34]. Although these two techniques are matured, there remain issues to be addressed. In photovoltaics, the conversion efficiency is close to a theoretical limit, and raw materials of silicon of high quality are about to run out. Of course alternative materials are being developed, but the cost and natural abundance are still issues. In the case of solar-thermal conversion, the conversion efficiency is not satisfactory except for some areas around the equators.

We have studied thermoelectric energy conversion using oxide materials, which are superior at high temperatures in air $[9,13]$. A serious drawback of thermoelectrics is poor efficiency [28]. A good thermoelectric material requires high electrical conductivity, large Seebeck coefficient, and low thermal conductivity at the same time, which is very difficult to be realized in real materials. In fact, reliable calculations of materials parameters do not give promising results. Since such calculations are done near equilibrium states, we hope that the thermoelectric performance may go beyond theoretical limitations in non-equilibrium states $[40,41]$. As such, we are trying to find ways to break through the poor efficiency by focusing non-equilibrium states. In this article, we show our preliminary results for two types of photo-excited thermoelectrics. One is the thermoelectrics using the photo-Seebeck effects (Fig. 1a), and the other is the thermoelectric energy conversion in a 


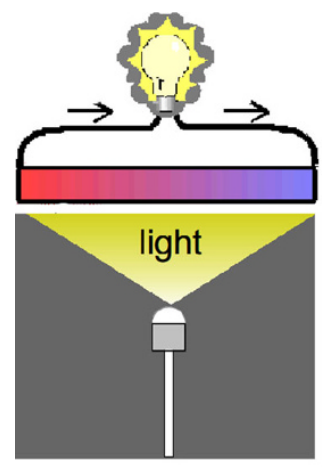

(a)

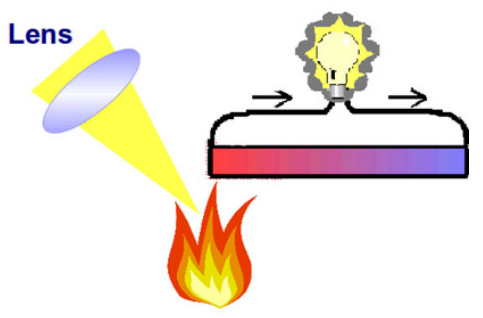

(b)
Fig. 1 Schematics of (a) power generation using photo-Seebeck effect and (b) power generation from focused light

large temperature gradient using solar light as a heat source (Fig. 1b). One may associate the two with photovoltaic and solar-thermal energy conversions.

To emphasize our originality, we will briefly summarize the preceding works. The photo-Seebeck effect was first reported by Tauc [31] in 1955, and was later examined in conventional semiconductors $[7,15]$. Note that the word "photo-thermoelectric" is confusing; it stood for the photoSeebeck effect before the 80 s, but is now used as photothermal energy conversion through the Seebeck effect (for example, see $[2,12])$. To our knowledge, our work is the first trial for the thermoelectric energy conversion using the photo-Seebeck effect. In contrast, the second trial shown in Fig. $1 \mathrm{~b}$ has been examined by many groups. Originally Telkes [32] examined the concept of solar thermoelectric generator in 1954. Naito et al. [18] designed a power converter using concentrated solar light and achieved a high temperature of 2,200 K in vacuum. Suter et al. [30] fabricated the solar thermoelectric generator and analyzed the efficiency and the maximum power. Fan et al. [3] reported an efficiency of $3 \%$ using commercially available Peltier modules. Thus, our originality lies on the fact that our device is made of single crystals of transition metal oxide, which can work at $800 \mathrm{~K}$ in air.

\section{Photo-Seebeck effect in oxide single crystals}

Although the first observation of the photo-Seebeck effect was reported in the mid 50s [31], there have been very few reports on the photo-Seebeck measurements since the 80 s. Thus, we had to newly establish measurement procedure with recently available equipments such as a light-emitting diode (LED). Here, we elaborate on the measurement and analysis details for the photo-Seebeck coefficient, which was not included in the published papers [17, 20].
Commercially available substrates were used as $\mathrm{ZnO}$ single crystals, and flux method was employed for making single crystals of $\mathrm{PbO}$. The photoconductivity of $\mathrm{ZnO}$ and $\mathrm{PbO}$ single crystals was measured with a two-probe method. The thermoelectric voltage of $\mathrm{ZnO}$ was measured with two-probe technique with several temperature difference and several photon intensities [20]. The resistance of $\mathrm{PbO}$ was too high to use the same measurement setup as in the case of $\mathrm{ZnO}$. Instead, the thermoelectric current was measured with several temperature difference and several photon intensities [17].

The photo-Seebeck effect is a change in the Seebeck coefficient with light illumination, which can be evaluated by comparing the thermoelectric voltage before and after illumination. However, the light illumination affects various properties at the same time; It causes the photovoltaic voltage at the contacts, increases the sample temperature, and changes the temperature difference. To be more quantitative, the measured thermoelectric voltage $V_{\mathrm{TE}}$ can be expressed by temperature difference $\Delta T$ at dark as

$V_{\mathrm{TE}}=S \Delta T+V_{0}$

where $S$ is the Seebeck coefficient at dark and $V_{0}$ is the offset voltage. When illuminated, the voltage can be written as

$V_{\mathrm{TE}}=(S+\delta S)(\Delta T+\delta T)+\left(V_{0}+\delta V_{0}\right)$,

where $\delta S$ is the photo-induced change in the Seebeck coefficient. $\delta T$ and $\delta V_{0}$ are the photo-induced temperature difference and photovoltaic component, respectively. Note that we measured the voltage in a cryostat (Quantum Design PPMS), where the sample temperature was strictly controlled.

The best way to measure the photo-Seebeck coefficient is to measure $V_{\mathrm{TE}}$ as a function of $\Delta T+\delta T$. When $V_{\mathrm{TE}}$ is found to be a linear function of $\Delta T+\delta T$, the photo-Seebeck coefficient of $S+\delta S$ and the photovoltaic component $V_{0}+\delta V_{0}$ are evaluated from the slope and the intercept, respectively. Figure 2 shows a typical example for the thermoelectric voltage with various photon intensities. The value of $I_{\text {LED }}$ represents the input current to an LED of 365 $\mathrm{nm}$ and $0 \mathrm{~mA}$ corresponds to the dark state. As is clearly shown, the slope systematically decreases with increasing $I_{\text {LED }}$, which means that the Seebeck coefficient decreases in magnitude with light illumination. This is reasonable because the light illumination generates carriers in the sample (photo-doping). Note that the intercept also changes with $I_{\text {LED }}$, which indicates a significant photovoltaic component induced by light illumination. We further comment that the temperature difference changes with $I_{\mathrm{LED}}$. This implies that the absorbed photon is converted into heat, and the heat flows through the sample to the heat bath. If the heat flow is completely homogeneous, the temperature 


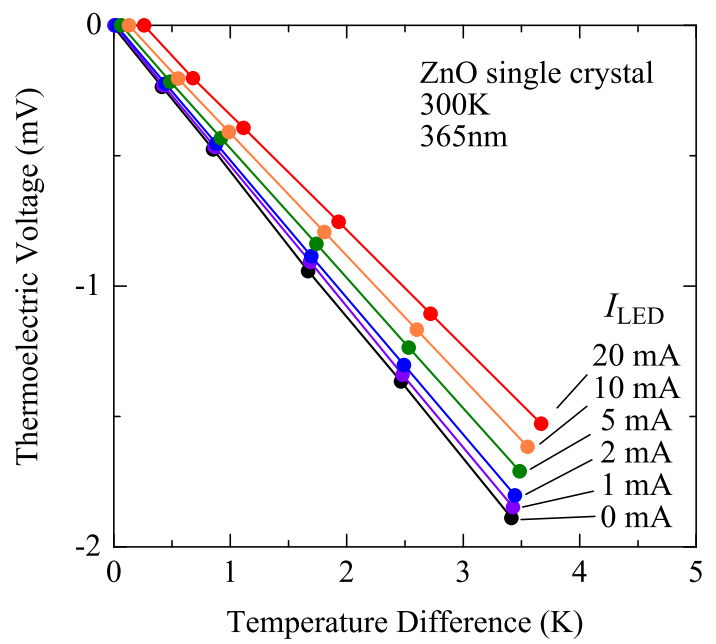

Fig. 2 Thermoelectric voltage in a $\mathrm{ZnO}$ single crystal plotted as a function of temperature difference. The LED current $I_{\mathrm{LED}}$ corresponds to the photon intensity

difference will be unchanged. Owing to unwanted asymmetry, a finite value of $\delta T$ is observed.

In the case of $\mathrm{PbO}$, the sample resistance is too high to measure $V_{\mathrm{TE}}$ because of the huge Johnson-Nyquist noise. Instead, the thermoelectric current $I_{\mathrm{TE}}$ was measured with a pico/femto-ampere meter as a function of temperature difference expressed by

$I_{\mathrm{TE}}=(S+\delta S)(\Delta T+\delta T) /(R-\delta R)+I_{0}$,

where $R$ is the sample resistance at dark and $\delta R$ is the photo-resistance of the sample. Thus, if the photo-resistance is measured in different runs, the thermoelectric voltage is evaluated as $(R-\delta R) I_{\mathrm{TE}}$. When $(R-\delta R) I_{\mathrm{TE}}$ is a linear function of $\Delta T+\delta T$, the photo-Seebeck coefficient is evaluated from the slope.

Figure 3 shows a typical experimental result in a $\mathrm{PbO}$ single crystal. The thermoelectric current $I_{\mathrm{TE}}$ is measured as a function of $\Delta T$ in Fig. 3a. With increasing $I_{\mathrm{LED}}$, the slope increases rapidly, which is mainly determined by photoconduction. Figure $3 \mathrm{~b}$ is the thermoelectric voltage plotted as a function of temperature difference, where the slope slightly changes with $I_{\mathrm{LED}}$, indicating photo-Seebeck effect in this oxide. Similarly to $\mathrm{ZnO}$, the results in Fig. 3 can be understood in terms of photo-doping. The type of the carrier is hole in $\mathrm{PbO}$, whereas it is electron in $\mathrm{ZnO}$. It should be noted that chemical doping is quite difficult in $\mathrm{PbO}$, and that light illumination is an effective tool for carrier doping in $\mathrm{PbO}$.

After a phenomenological analysis based on the twolayer model, we have obtained the intrinsic photo-Seebeck coefficient and the intrinsic values of photo-doped carrier density $[17,20]$. We find a maximum value for the photodoped electron density is of the order of $10^{19} \mathrm{~cm}^{-3}$, which is nearly the same value of the optimally doped

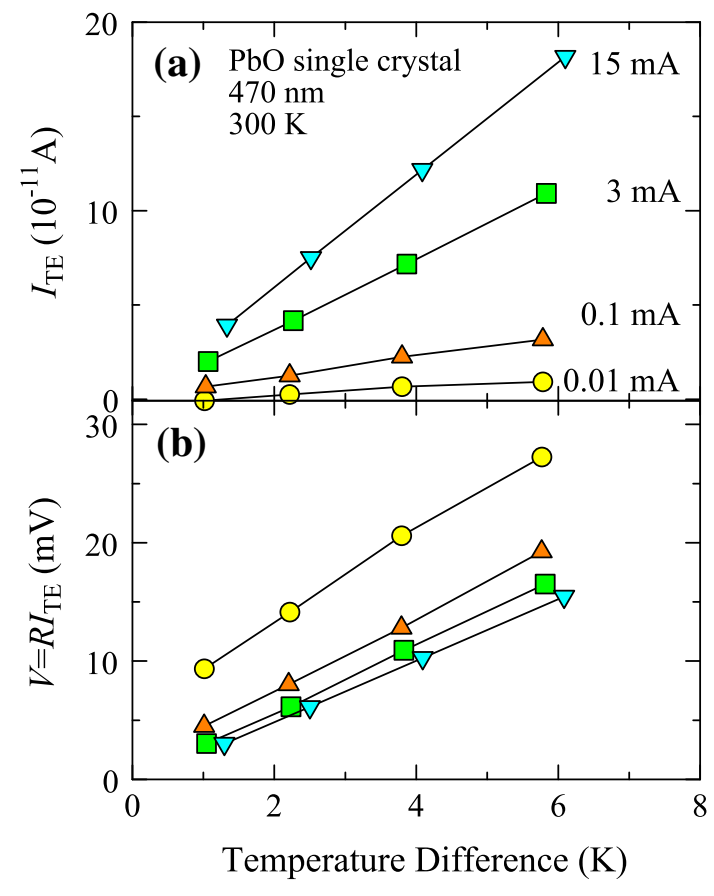

Fig. 3 (a) Thermoelectric current $I_{\mathrm{TE}}$ and (b) thermoelectric voltage $\mathrm{RI}_{\mathrm{TE}}$ in a $\mathrm{PbO}$ single crystal plotted as a function of temperature difference. The numbers in $\mathrm{mA}$ represent the LED current $I_{\mathrm{LED}}$ corresponding to the photon intensity

thermoelectric semiconductors [28]. Thus, we can conclude that the ultraviolet LED can be an alternative carrier source. In the case of $\mathrm{PbO}$, a maximum value is of the order of $10^{18} \mathrm{~cm}^{-3}$, one-order of magnitude smaller than in the case of $\mathrm{ZnO}$.

\section{Thermoelectric uni-couple made of single-crystal oxides}

Since the discovery of good thermoelectric properties in the layered cobalt oxide $\mathrm{Na}_{x} \mathrm{CoO}_{2}$ [33], oxide thermoelectrics has been explored extensively. The layered cobalt oxides are now ready to be applied for thermoelectric power generation, and a prototype power generator is now commercially available at a venture company [1]. All the layered cobalt oxides show good thermoelectric performance at high temperatures. Above $700 \mathrm{~K}$, the thermoelectric performance exceeds a qualifying value (the dimensionless figure of merit larger than unity) in singlecrystal samples $[5,6,25]$, while it remains a somewhat lower value in ceramic samples [11, 19, 37, 39]. Thus, a device using single crystals is expected to show a better performance than ceramic samples.

We have made a trial device of a uni-couple consisting of single-crystal $\mathrm{Bi}_{2} \mathrm{Sr}_{2} \mathrm{Co}_{2} \mathrm{O}_{y}$ (p-type) and single-crystal $\mathrm{CaMnO}_{3}$ (n-type). Single crystals were grown through a floating-zone technique, and the $p-n$ junction was made 


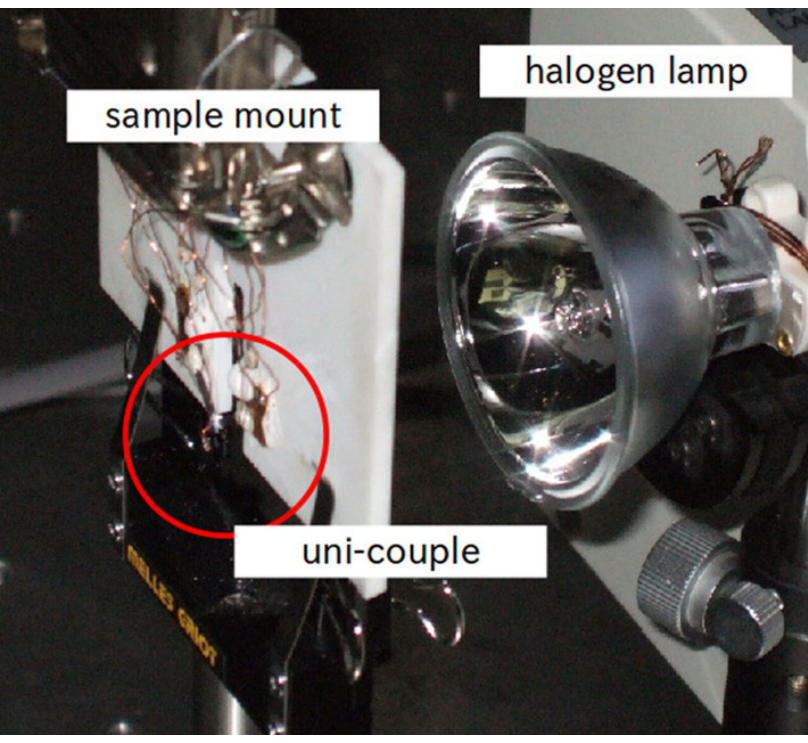

Fig. 4 Power curve measurement setup using halogen lamp as a heat source

using silver paint. The dimensions were $1.4 \times 1.2 \times 9.4$ $\mathrm{mm}^{3}$ for $p$-type and $1.2 \times 1.1 \times 11 \mathrm{~mm}^{3}$ for $n$-type. The current-voltage characteristics were measured with a home-made characterization system. A focused halogen light was used as a heat source, and three platinum resistance thermometers were attached at the ends of the device (one at the hot junction and the other two at the cold edges). Figure 4 shows a photographic image of our measurement setup. The device supported on a machinable ceramic plate is set at the focal point of the halogen lamp.

Figure 5 shows typical power curves of the singlecrystal uni-couple. Since the device design is not yet optimized, the maximum power output is only $1.5 \mathrm{~mW}$. Although both $p$ - and $n$-legs are made of single crystals, the thermoelectric properties are not yet optimized either. In addition, this measurement includes the contact resistance and circuit resistance, which underestimates the power output.

Let us compare the device performance with other oxide devices. Table 1 lists the oxide device performance reported so far. The materials, the operating temperature difference $\Delta T$, and the number of pairs $N$ are different from one to another. The maximum output per pair $P_{p}$ in our device is comparable with some devices.

One characteristic feature of this device is that a large temperature difference is generated across the sample. Figure 6a shows the hot-side temperature $T_{\mathrm{H}}$ and the coldside temperature $T_{\mathrm{C}}$ of the uni-couple plotted as a function of the input voltage of the halogen lamp $V_{\text {light }}$. With increasing $V_{\text {light }}, T_{\mathrm{H}}$ increases monotonically, while the two $T_{\mathrm{C}}$ 's almost stay near room temperature. At a maximum voltage of $14 \mathrm{~V}$, the temperature difference reaches $500 \mathrm{~K}$.

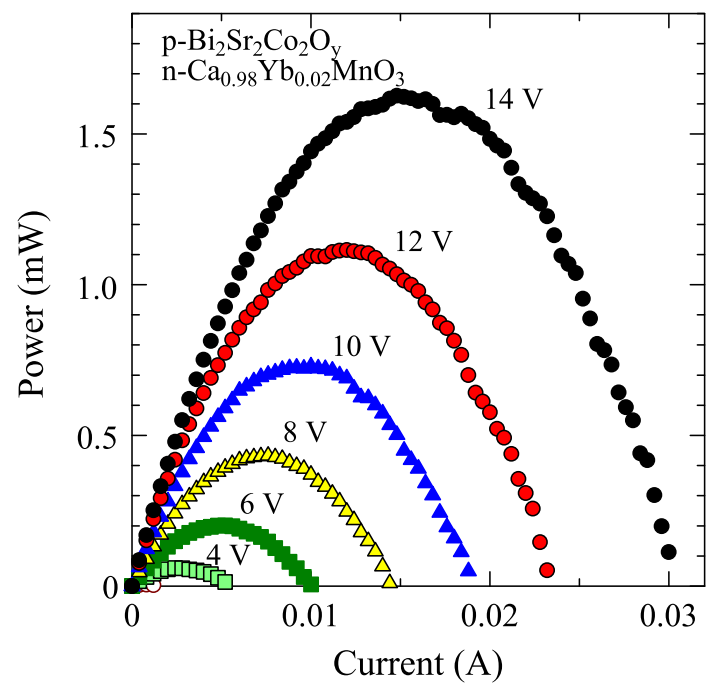

Fig. 5 Power curves of the uni-couple device made of single-crystal oxides. The numbers in volt represent the input voltage for the halogen lamp

Table 1 Comparison with oxide thermoelectric modules

\begin{tabular}{|c|c|c|c|c|c|c|}
\hline Reference & Materials & $N$ & $\begin{array}{l}\Delta T \\
(\mathrm{~K})\end{array}$ & $\begin{array}{l}V_{\mathrm{oc}} \\
(\mathrm{mV})\end{array}$ & $\begin{array}{l}P_{\max } \\
(\mathrm{mW})\end{array}$ & $\begin{array}{l}P_{p} \\
(\mathrm{~mW})\end{array}$ \\
\hline $\begin{array}{l}\text { Matsubara et al. } \\
\text { [16] }\end{array}$ & $\begin{array}{c}\mathrm{CaGdCoO} / \\
\mathrm{CaMnO}\end{array}$ & 8 & 773 & 988 & 63.5 & 7.9 \\
\hline Urata et al. [36] & $\mathrm{CaCoO} / \mathrm{CaMnO}$ & 8 & 973 & 700 & 340 & 42.5 \\
\hline Shin et al. [26] & $\begin{array}{r}\mathrm{NaCoCuO} / \\
\mathrm{BaSrPbO}\end{array}$ & 1 & 506 & 140 & 12.0 & 12.0 \\
\hline Ono et al. [21] & $\begin{array}{l}\mathrm{NaCoO} / \\
\mathrm{CaLaMnO}\end{array}$ & 2 & 166 & 63 & 2.3 & 1.15 \\
\hline Hayashi et al. [8] & $\begin{array}{l}\mathrm{LaSrCuO} / \\
\mathrm{NdCeCuO}\end{array}$ & 25 & 360 & 1,300 & 26.3 & 1.05 \\
\hline Reddy et al. [23] & $\begin{array}{l}\mathrm{CaCoO} / \\
\mathrm{CaSmMnO}\end{array}$ & 2 & 925 & 400 & 31.5 & 17.0 \\
\hline Tomes et al. [35] & $\begin{array}{l}\mathrm{LaSrCuO} / \\
\mathrm{CaMnNbO}\end{array}$ & 2 & 622 & 46 & 88.8 & 44.4 \\
\hline $\begin{array}{l}\text { Inagoya et al. } \\
\text { [10] }\end{array}$ & $\begin{array}{l}\mathrm{NdCaCoO} / \\
\mathrm{LaCoMnO}\end{array}$ & 10 & 170 & 1,010 & 10.8 & 1.08 \\
\hline This work & $\begin{array}{l}\text { BiSrCoO/ } \\
\text { CaYbMnO }\end{array}$ & 1 & 500 & 200 & 1.6 & 1.6 \\
\hline
\end{tabular}

The number of pairs $N$, the temperature difference $\Delta T$, the opencircuit voltage $V_{\mathrm{oc}}$, and the maximum power $P_{\max }$ are listed. The maximum power per pair $P_{p}=P_{\max } / N$ is also listed

Since the device length is approximately $1 \mathrm{~cm}$, the temperature gradient reaches a large value of $500 \mathrm{~K} / \mathrm{cm}$ by aircooling without blower.

Owing to the large temperature difference, thermoelectric parameters change within the device though their temperature dependence. Snyder and Ursell [29] have found the exact expression for the conversion efficiency that is valid even in such conditions. Surprisingly, the efficiency is determined only by the values at the high and 


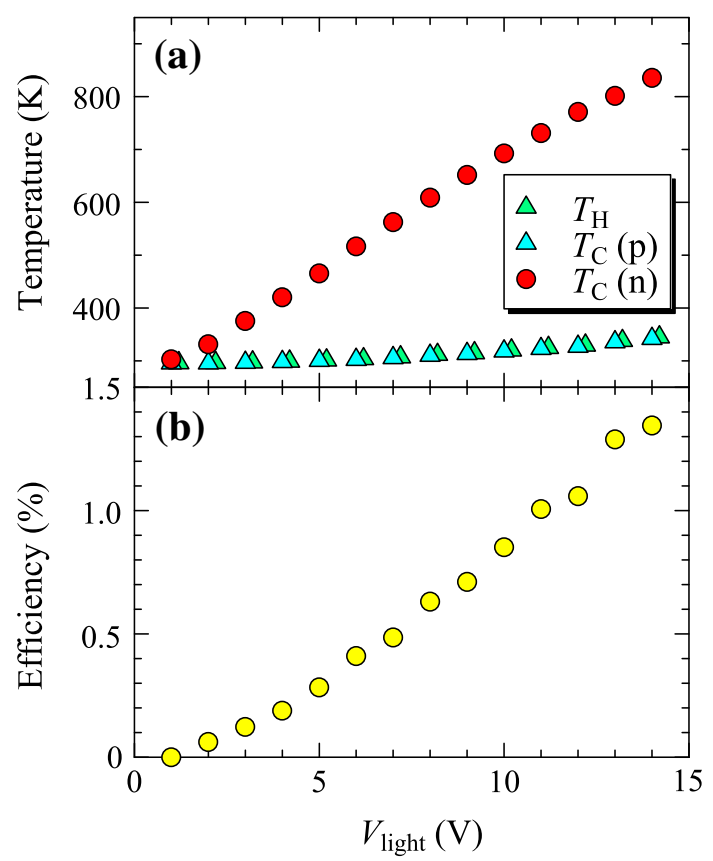

Fig. 6 (a) The temperatures at the hot edge $T_{\mathrm{H}}$ and the temperatures at the cold edges $T_{\mathrm{C}}$ 's (b) the conversion efficiency calculated by Eq. (4) plotted as a function of the input voltage for the halogen lamp $V_{\text {light }}$

low temperature edges of the device. A concrete form of the expression is given by

$\eta=1-\frac{S_{\mathrm{C}} T_{\mathrm{C}}+1 / u_{\mathrm{C}}}{S_{\mathrm{H}} T_{\mathrm{H}}+1 / u_{\mathrm{H}}}$,

where the subscripts of $\mathrm{H}$ and $\mathrm{C}$ represent the values at the hot and cold edges. The quantity of $u \equiv j / \kappa \nabla T$ is called the relative current density, which is the ratio of the electrical current density to the heat flux density by thermal conduction.

The temperatures $\left(T_{\mathrm{H}}\right.$ and $\left.T_{\mathrm{C}}\right)$ at the cold and hot edges were monitored in the power curve measurements. The Seebeck coefficients $\left(S_{\mathrm{H}}\right.$ and $\left.S_{\mathrm{C}}\right)$ at the cold and hot edges were known by measuring the Seebeck coefficients of $p$ and $n$-type crystals in advance. To calculate the relative current density, the resistivity and the thermal conductivity are necessary. The resistivity values were measured for the prepared crystals, and the thermal conductivity data were taken from the literature $[4,24]$. Figure $6 \mathrm{~b}$ shows the conversion efficiency plotted as a function of $V_{\text {light }}$. The maximum efficiency is a reasonably large value of $1.4 \%$ in spite of the fact that all the conditions were far from ideal. The present measurement is a first step for this kind of thermoelectric power generator, and we notice many issues to improve the device performance. Kraemer et al. [14] have reported an efficiency of $4.6 \%$ in a solar thermoelectric generators using $\mathrm{Bi}_{2} \mathrm{Te}_{3}$-based device. Although the device concept is completely different from our device, we preliminarily obtained a similar efficiency in another modified uni-couple (not shown).

An advantage of oxide device is seen in the smooth evolution of the power curves in Fig. 5. This implies that the device resistance is almost constant from 300 to $800 \mathrm{~K}$, and the device is sufficiently stable at $800 \mathrm{~K}$ in air. Considering that the surface temperature at the focal point on the device is much higher, we think that this experiment demonstrates the high-temperature stability of oxide devise in air.

We are considering a possibility to use this type of device to the black-body radiation from high-temperature furnace. Recalling that the sun is a black body of $6,000 \mathrm{~K}$, we can use black-body radiation from other hot sources as well. For example, a black body of 1,000 $\mathrm{K}$ will give a large photon flux of $57 \mathrm{~kW} / \mathrm{m}^{2}$, being 50 times larger than that of the sun. Heat is difficult to control; It is hardly stored, transported or accumulated at our disposal, but electromagnetic wave can be done so.

\section{Summary and future prospects}

In this article, we have reported our attempts to find a better thermoelectric power generation using light. One approach is the thermoelectrics using the photo-Seebeck effect. We have shown the $n$-type photo-Seebeck effect in $\mathrm{ZnO}$ and the $p$-type one in $\mathrm{PbO}$. Although the thermoelectric energy conversion is not yet done, the photo-doping gives reasonably good thermoelectric parameters. The second approach is the thermoelectric energy conversion using focused light as a heat source. The device structure and thermoelectric materials are not yet optimized, and the efficiency is $1.4 \%$ at the present stage. Nevertheless, we have successfully shown the high-temperature stability of the oxide device, and also shown that the oxide thermoelectric uni-couple can be made from single crystals. The former approach uses ultraviolet light for a doping tool, while the latter uses infrared light as a heat source. Since these two lights are not used in Si-based solar cells, our two approaches can live with solar cells to increase the total energy conversion efficiency. Actually, Park et al. [22] and Wang et al. [38] already have combined an existing photovoltaic cell with a thermoelectric module. We look forward to developing a new device making full use of solar energy by combining photovoltaics with thermoelectrics, even though we cannot see the concrete shape at the moment.

Acknowledgments We would like to thank A. Horikawa for her tenacious efforts to establish measurement procedure for the photoSeebeck effect. We also wish to thank Y. Yasui, H. Taniguchi, M. Fujita, and Y. Shiraishi for close collaboration of this work. We also appreciate R. Funahashi and H. Ohta for fruitful discussion. 
Open Access This article is distributed under the terms of the Creative Commons Attribution License which permits any use, distribution, and reproduction in any medium, provided the original author(s) and the source are credited.

\section{References}

1. TES New Energy corporation (2010). http://tes-ne.com/English/ 01_home_e.html

2. Basko, D.: A photothermoelectric effect in graphene. Science 334(6056), 610-611 (2011). doi:10.1126/science.1214560

3. Fan, H., Singh, R., Akbarzadeh, A.: Electric power generation from thermoelectric cells using a solar dish concentrator. J. Electr. Mater. 40(5), 1311-1320 (2011). doi:10.1007/s11664-011-1625-x

4. Flahaut, D., Mihara, T., Funahashi, R., Nabeshima, N., Lee, K., Ohta, H., Koumoto, K.: Thermoelectrical properties of A-site substituted $\mathrm{Ca}_{1-x} \mathrm{Re}_{x} \mathrm{MnO}_{3}$ system. J. Appl. Phys. 100(8), 084911 (2006). doi:10.1063/1.2362922

5. Fujita, K., Mochida, T., Nakamura, K.: High-temperature thermoelectric properties of $\mathrm{Na}_{x} \mathrm{CoO}_{2-\delta}$ single crystals. Jpn. J. Appl. Phys. 40(Part 1, No. 7), 4644-4647 (2001). doi:10.7567/JJAP.40.4644

6. Funahashi, R., Shikano, M.: $\mathrm{Bi}_{2} \mathrm{Sr}_{2} \mathrm{Co}_{2} \mathrm{O}_{y}$ whiskers with high thermoelectric figure of merit. Appl. Phys. Lett. 81(8), 1459-1461 (2002). doi: 10.1063/1.1502190

7. Harper, J.G., Matthews, H.E., Bube, R.H.: Two-carrier photothermoelectric effects in GaAs. J. Appl. Phys. 41(7), 3182-3184 (1970). doi:10.1063/1.1659387

8. Hayashi, S.F., Nakamura, T., Kageyama, K., Takagi, H.: Monolithic thermoelectric devices prepared with multilayer cofired ceramics technology. Jpn. J. Appl. Phys. 49(9), 096505 (2010). doi:10.7567/JJAP.49.096505

9. He, J., Liu, Y., Funahashi, R.: Oxide thermoelectrics: the challenges, progress, and outlook. J. Mater. Res. 26(15), 1762-1772 (2011). doi:10.1557/jmr.2011.108

10. Inagoya, A., Sawaki, D., Horiuchi, Y., Urata, S., Funahashi, R., Terasaki, I.: Thermoelectric module made of perovskite cobalt oxides with large thermopower. J. Appl. Phys. 110(12), 123712 (2011). doi:10.1063/1.3671070

11. Ito, M., Nagira, T., Furumoto, D., Katsuyama, S., Nagai, H.: Synthesis of $\mathrm{Na}_{x} \mathrm{Co}_{2} \mathrm{O}_{4}$ thermoelectric oxides by the polymerized complex method. Scripta Mater. 48(4), 403-408 (2003). doi: 10. 1016/S1359-6462(02)00443-8

12. Kasymakhunova, A., Nabiev, M.: Photothermoelectric converters of concentrated radiation. Tech. Phys. Lett. 29(3), 253-255 (2003). doi:10.1134/1.1565650

13. Koumoto, K., Wang, Y., Zhang, R., Kosuga, A., Funahashi, R.: Oxide thermoelectric materials: a nanostructuring approach. Ann. Rev. Mater. Res. 40(1), 363-394 (2010). doi:10.1146/annurevmatsci-070909-104521

14. Kraemer, D., Poudel, B., Feng, Hp, Caylor, J.C., Yu, B., Yan, X., Ma, Y., Wang, X., Wang, D., Muto, A., McEnaney, K., Chiesa, M., Ren, Z., Chen, G.: High-performance flat-panel solar thermoelectric generators with high thermal concentration. Nat. Mater. 10, 532-538 (2011). doi:10.1038/nmat3013

15. Kwok, H.B., Bube, R.H.: Thermoelectric and photothermoelectric effects in semiconductors: CdS single crystals. J. Appl. Phys. 44(1), 138-144 (1973). doi:10.1063/1.1661848

16. Matsubara, I., Funahashi, R., Takeuchi, T., Sodeoka, S., Shimizu, T., Ueno, K.: Fabrication of an all-oxide thermoelectric power generator. Appl. Phys. Lett. 78(23), 3627-3629 (2001). doi:10.1063/1. 1376155

17. Mondal, P.S., Okazaki, R., Taniguchi, H., Terasaki, I.: Photoseebeck effect in tetragonal $\mathrm{PbO}$ single crystals. J. Appl. Phys. 114(17), 173710 (2013). doi:10.1063/1.4829460
18. Naito, H., Kohsaka, Y., Cooke, D., Arashi, H.: Development of a solar receiver for a high-efficiency thermionic/thermoelectric conversion system. Sol. Energy 58(4-6), 191-195 (1996). doi:10. 1016/S0038-092X(96)00084-9

19. Ohtaki, M., Nojiri, Y., Maeda, E.: Improved thermoelectric performance of sintered $\mathrm{NaCo}_{2} \mathrm{O}_{4}$ with enhanced 2-dimensional microstructure. In: Rowe, M. (ed.) Proceedings of The 19th International Conference on Thermoelectrics (ICT2000), pp. 190-195. Babrow, Cardiff (2000)

20. Okazaki, R., Horikawa, A., Yasui, Y., Terasaki, I.: Photo-seebeck effect in ZnO. J. Phys. Soc. Jpn. 81(11), 114722 (2012). doi:10. 1143/JPSJ.81.114722

21. Ono, Y., Kimura, D., Kawano, S.H.O., Watanabe, R., Kajitani, T.: Fabrication and performance of an oxide thermoelectric power generator. In: Proceedings of The 21st International Conference on Thermoelectrics (ICT2002), pp. 454-457. IEEE (2002)

22. Park, K.T., Shin, S.M., Tazebay, A.S., Um, H.D., Jung, J.Y., Jee, S.W., Oh, M.W., Park, S.D., Yoo, B., Yu, C., Lee, J.H.: Lossless hybridization between photovoltaic and thermoelectric devices. Sci. Rep. 3, 2123 (2013). doi:10.1038/srep02123

23. Reddy, E.S., Noudem, J.G., Hebert, S., Goupil, C.: Fabrication and properties of four-leg oxide thermoelectric modules. J. Phys. D 38(19), 3751-3755 (2005). doi:10.1088/0022-3727/38/19/026

24. Satake, A., Tanaka, H., Ohkawa, T., Fujii, T., Terasaki, I.: Thermal conductivity of the thermoelectric layered cobalt oxides measured by the Harman method. J. Appl. Phys. 96(1), 931-933 (2004). doi:10.1063/1.1753070

25. Shikano, M., Funahashi, R.: Electrical and thermal properties of single-crystalline $\left(\mathrm{Ca}_{2} \mathrm{CoO}_{3}\right)_{0.7} \mathrm{CoO}_{2}$ with a $\mathrm{Ca}_{3} \mathrm{Co}_{4} \mathrm{O}_{9}$ structure. Appl. Phys. Lett. 82(12), 1851-1853 (2003). doi: 10.1063/1. 1562337

26. Shin, W., Murayama, N., Ikeda, K., Sago, S., Terasaki, I.: Thermoelectric device of $\mathrm{Na}(\mathrm{Co}, \mathrm{Cu})_{2} \mathrm{O}_{4}$ and $(\mathrm{Ba}, \mathrm{Sr}) \mathrm{PbO}_{3}$. J. Cer. Soc. Jpn. 110(8), 727-730 (2002). doi: 10.2109/jcersj.110.727

27. Singh, G.: Solar power generation by PV (photovoltaic) technology: a review. Energy 53, 1-13 (2013). doi:10.1016/j.energy. 2013.02.057

28. Snyder, G.J., Toberer, E.S.: Complex thermoelectric materials. Nat. Mater. 7, 105-114 (2008). doi:10.1038/nmat2090

29. Snyder, G.J., Ursell, T.S.: Thermoelectric efficiency and compatibility. Phys. Rev. Lett. 91(14), 148301 (2003). doi:10.1103/ PhysRevLett.91.148301

30. Suter, C., Tomes, P., Weidenkaff, A., Steinfeld, A.: Heat transfer and geometrical analysis of thermoelectric converters driven by concentrated solar radiation. Materials 3(4), 2735-2752 (2010). doi:10.3390/ma3042735

31. Tauc, J.: The thermal photo-electric phenomenon in semi-conductors. Czech. Phys. J. 5(4), 528-535 (1955). doi:10.1007/ BF01687219

32. Telkes, M.: Solar thermoelectric generators. J. Appl. Phys. 25(6), 765-777 (1954). doi:10.1063/1.1721728

33. Terasaki, I., Sasago, Y., Uchinokura, K.: Large thermoelectric power in $\mathrm{NaCo}_{2} \mathrm{O}_{4}$ single crystals. Phys. Rev. B 56(20), R12685R12687 (1997). doi: 10.1103/PhysRevB.56.R12685

34. Thirugnanasambandam, M., Iniyan, S., Goic, R.: A review of solar thermal technologies. Renew. Sustain. Energy Rev. 14(1), 312-322 (2010). doi:10.1016/j.rser.2009.07.014

35. Tomes, P., Trottmann, M., Suter, C., Aguirre, M.H., Steinfeld, A., Haueter, P., Weidenkaff, A.: Thermoelectric oxide modules (TOMs) for the direct conversion of simulated solar radiation into electrical energy. Materials 3(4), 2801-2814 (2010). doi:10.3390/ ma3042801

36. Urata, S., Funahashi, R., Mihara, T., Kosuga, A., Sodeoka, S., Tanaka, T.: Power generation of a p-type $\mathrm{Ca}_{3} \mathrm{Co}_{4} \mathrm{O}_{9} /$ n-type $\mathrm{CaMnO}_{3}$ module. Int. J. Appl. Cer. Technol. 4(6), 535-540 (2007). doi: 10.1111/j.1744-7402.2007.02173.x 
37. Van Nong, N., Pryds, N., Linderoth, S., Ohtaki, M.: Enhancement of the thermoelectric performance of $p$-type layered oxide $\mathrm{Ca}_{3} \mathrm{Co}_{4} \mathrm{O}_{9+d}$ through heavy doping and metallic nanoinclusions. Adv. Mater. 23(21), 2484-2490 (2011). doi: 10.1002/adma.201004782

38. Wang, N., Han, L., He, H., Park, N.H., Koumoto, K.: A novel high-performance photovoltaic-thermoelectric hybrid device. Energy Environ. Sci. 4(9), 3676-3679 (2011). doi:10.1039/ clee01646f

39. Wang, Y., Sui, Y., Cheng, J., Wang, X., Su, W.: Comparison of the high temperature thermoelectric properties for Ag-doped and
Ag-added $\mathrm{Ca}_{3} \mathrm{Co}_{4} \mathrm{O}_{9}$. J. Alloys Comp. 477(1-2), 817-821 (2009). doi: 10.1016/j.jallcom.2008.10.162

40. Whitney, R.S.: Nonlinear thermoelectricity in point contacts at pinch off: a catastrophe aids cooling. Phys. Rev. B 88(6), 064302 (2013). doi:10.1103/PhysRevB.88.064302

41. Zebarjadi, M., Esfarjani, K., Shakouri, A.: Nonlinear peltier effect in semiconductors. Appl. Phys. Lett. 91(12), 104122 (2007). doi:10.1063/1.2785154 